\title{
Synthesis and characterization of a new Cd-based
}

\section{Metal-Organic Framework isostructural with MOF-}

\section{4 / CPO-27 materials}

\author{
Manuel Díaz-García, and Manuel Sánchez-Sánchez*
}

Instituto de Catálisis y Petroleoquímica, ICP-CSIC, C/ Marie Curie 2, 28049 Madrid, Spain

*To whom correspondence should be addressed:

Dr. Manuel Sánchez-Sánchez. E-mail addresses: manuel.sanchez@icp.csic.es

Telephone: +34-915854795. Fax: +34-915854760.

ABSTRACT. A new M-MOF-74 material, with M being cadmium, is described. The so-called Cd-MOF-74 material was prepared under solvothermal conditions using cadmium acetate as metal source. It was characterized by a variety of physic-chemical techniques including PXRD, TGA, $\mathrm{N}_{2}$ adsorption-desorption isotherms, SEM, IR and multinuclear solid-state NMR. Although the quality and/or the size of the afforded crystals did not allow us solving the structure by single-crystal X-ray diffraction, the information given by different characterization techniques is enough to confirm that the sample is indeed a Cd-based MOF-74-like material. Fingerprint-based PXRD and FTIR techniques showed a good matching between the Cd-MOF-74 and the wellknown Zn-MOF-74 samples. Likewise, TGA and $\mathrm{N}_{2}$ adsorption-desorption at $196^{\circ} \mathrm{C}$ gave expected values of $\mathrm{Cd} /$ /inker ratio and textural properties, respectively. SEM certified the 
homogeneity of crystals and the absence of impurities. Among the divalent metal ions capable to produce MOF-74 materials, $\mathrm{Cd}^{2+}$ presents some singularities: (i) it is the first metal of the second transition metal row able to led to a M-MOF-74 material; (ii) due to reasonably good magnetic properties of the diamagnetic non-quadrupolar ${ }^{113} \mathrm{Cd}$ and ${ }^{111} \mathrm{Cd}$ nuclei, metal-adsorbate interactions can be studied from a metal point of view by means of NMR techniques.

KEYWORDS. MOF-74; CPO-27-Cd; unit cell dimensions; etb topology; ${ }^{113} \mathrm{Cd} \mathrm{NMR}$.

\section{INTRODUCTION}

Among the families of porous materials, that of the Metal-Organic Frameworks (MOFs) is certainly the most emerging one for so many applications[1,2]. Apart from the exceptional textural properties reached by some MOFs, widely overcome these of conventional microporous materials such as zeolites, the MOF materials family has some properties unprecedented or at least uncommonly found in any other known porous materials, such as network catenation[3], flexibility[4], structural chirality[5], or exposed and unsaturated metal centers[6]. Some of the most deeply studied MOF materials having open metal sites are these of the CPO-27-M family, also known as MOF-74 or simply $\mathrm{M}_{2}$ (dhtp) or $\mathrm{M}_{2}$ (dohbdc), where dhtp or dohbc represent the organic linker 2,5-dihydroxybenzyldicarboxylate. Several reasons are behind the interest in this particular isostructural MOF materials: (i) the already-mentioned presence of open metal sites; (ii) their versatility of being prepared with different divalent ions $(\mathrm{Mg}[7,8], \mathrm{Mn}[9], \mathrm{Fe}[10]$, $\mathrm{Co}[11], \mathrm{Ni}[12]$ and $\mathrm{Zn}[13,14]$ ) or with a mixture of them at any extension[15]; (iii) their thermal and chemical stability under ambient conditions and in presence of water[16]; (iv) the possibility of being prepared at room temperature[17]; v) their high adsorption heat for highly-demanded 
gases such as $\mathrm{H}_{2}[18]$ and $\mathrm{CO}_{2}[19]$; and vi) their high efficiency in the separation of molecules of similar size[20]. A proof of the attention paid to these MOFs can be deduced from three papers published in the last few months, which expand both the compositional diversity and the potential applications of MOF-74-like materials: (i) a new Cu-based MOF-74[21], (ii) a Mnbased MOF-74-like MOF using a 2,5-dithiolbenezenedicarboxylate as linker (that is, -SH groups instead of the $-\mathrm{OH}$ groups found in the dhtp linker) with potential applications in conductivity[22]; and (iii) the series of isoreticular IRMOF-74 materials prepared with organic linkers similar to dhtp but having different length and/or functionalization, leading to the IRMOF-74-XI material [23] having the largest pore (near $10 \mathrm{~nm}$ of diameter) ever reported in MOFs.

MOF-74 materials were firstly described with $\mathrm{Zn}$ as metal[13]. In spite of the undoubted interest for some other members of the M-MOF-74 family, Zn seems to have a particular propensity to lead these materials, what has been made clear by its choice to develop the IRMOF-74 materials[23]. On the other hand, chemical similarities between $\mathrm{Zn}$ and $\mathrm{Cd}$ are wellknown, as they belong to the same group in Periodic Table. Such behavior is not an exception and some isostructural MOF-based materials prepared with either $\mathrm{Zn}$ or $\mathrm{Cd}[24-31]$ have been described, in spite of their quite different ionic sizes. Therefore, the choice of $\mathrm{Cd}$ to try to extend the M-MOF-74 family to the second transition metal row seems reasonable. Additionally, the acceptably good NMR properties of the ${ }^{111} \mathrm{Cd}$ and ${ }^{113} \mathrm{Cd}$ would open the possibility of studying the interaction between open metal sites and adsorbates / reactants, so far rather limited[32] by either the paramagnetic character of some metal ions M or by the low NMR sensitivity of some other M isotopes. 


\section{EXPERIMENTAL}

\subsection{Synthesis of the Cd-MOF-74 material}

Cd-MOF-74 material was synthesized by solvothermal treatment of a clear solution of molar composition 2.6 Cd : $1.0 \mathrm{dhtp}: 268 \mathrm{DMF}$, where dhtp represents the organic linker 2,5dihydroxyterphthalic acid and DMF is the solvent N,N,-dimethylformamide. In a typical procedure, a clear yellow solution of $0.200 \mathrm{~g}$ of dhtp (Aldrich) in $5.0 \mathrm{~g}$ of DMF (Aldrich) was added over other clear solution of $0.699 \mathrm{~g}$ of cadmium acetate dihydrate (Aldrich) in $15.0 \mathrm{~g}$ of DMF. The resultant clear solution was solvothermally treated at $125^{\circ} \mathrm{C}$ for 20 hours. A light yellow solid was obtained after such treatment, recovered by centrifugation and washed first with $20 \mathrm{~mL}$ of DMF and then two more times with $20 \mathrm{~mL}$ of methanol. The solid was kept submerged in methanol for 6 days; during these days, methanol covering the MOF sample was changed three times by fresh methanol.

For comparison purposes, a conventional Zn-MOF-74 material was prepared according to a described procedure[13].

\subsection{Characterization techniques}

Powder X-ray diffraction (XRD) patterns were acquired with a Philips X'PERT diffractometer using $\mathrm{Cu} \mathrm{K} \alpha$ radiation. Unit cell parameters were estimated with the XRD software CELREF from PXRD patterns registered with $2 \theta$ steps of $0.01^{\circ}$ and radiation time of $100 \mathrm{~s}$ per point. The ten most intense non-overlapped reflections were selected and adjusted to the space group R-3 (no. 148) starting from the unit cell parameters determined by single-crystal X-ray diffraction of a Zn-MOF-74 material[13]. In order to get accurate $2 \theta$ values of the reflections, an internal 
standard of Si was physically mixed with the MOF-74 samples, and its reflection 111 (at $2 \theta$ of $28.4^{\circ}$ ) of the former was taken as reference of $2 \theta$ position for the MOF-74 XRD reflections. Theoretical PXRD pattern of Zn-MOF-74 was generated by Mercury software from the CIF file reported elsewhere[13]. Theoretical pattern of Cd-MOF-74 was obtained by Materials Studio software starting from the structure of $\mathrm{Zn}-\mathrm{MOF}-74$ and changing the $\mathrm{Zn}$ atoms by $\mathrm{Cd}$ ones, and considering the unit cell parameters estimated from the analysis of experimental PXRD pattern of Cd-MOF-74 sample. The CIF file of the Cd-MOF-74 structure, as well as that of Zn-MOF-74 published elsewhere[13], could be find in the Supporting Information.

Attenuated total reflection-Fourier transform infrared (ATR-FTIR) spectra were recorded using a Thermo Nicolet Nexus 670 FTIR spectrometer equipped with a SensIR Technologies DurasamplIR horizontal ATR accessory and a liquid nitrogen-cooled MCT detector.

Thermogravimetric analyses (TGA) were registered in a Perkin-Elmer TGA7 instrument. TG analyses were carried out at a heating rate of $20^{\circ} \mathrm{C} / \mathrm{min}$ under air flow.

Nitrogen adsorption/desorption isotherms were measured at $-196^{\circ} \mathrm{C}$ in a Micromeritics ASAP 2020 device. Before the measurement, the previously calcined sample was degassed at $100{ }^{\circ} \mathrm{C}$ under high vacuum for at least 18 hours. Surface areas were estimated by BET method. Micropore and external surface areas were estimated by applying t-plot method.

Scanning Electron Microscopy (SEM) studies were carried out in an Ultra-high Resolution FEI-NOVA NanoSEM 230 FESEM instrument.

All Nuclear Magnetic Resonance (NMR) spectra were acquired in a Bruker AV-400-WB spectrometer operating at $100.61 \mathrm{MHz}$ for ${ }^{13} \mathrm{C}$ NMR spectra and at $88.80 \mathrm{MHz}$ for ${ }^{113} \mathrm{Cd} \mathrm{NMR}$ spectra, under magic-angle spinning (MAS) technique. A 4-mm probe and a spinning rate of 10 $\mathrm{kHz}$ were used during the acquisition of all spectra. ${ }^{1} \mathrm{H}$ to ${ }^{13} \mathrm{C}$ cross-polarization MAS NMR 
spectra were acquired applying a $\pi / 2$ pulse of $3 \mu$ s over the ${ }^{1} \mathrm{H}$ channel, using a contact time of $3.5 \mathrm{~ms}$ and a pulse delay of $5 \mathrm{~s}$. In the acquisition of ${ }^{1} \mathrm{H}$ to ${ }^{113} \mathrm{Cd}$ cross-polarization MAS NMR spectra, the ${ }^{1} \mathrm{H} \pi / 2$ pulse length was $2.8 \mu \mathrm{s}$, the contact time of $9.0 \mathrm{~ms}$ and the pulse delay of $5 \mathrm{~s}$.

${ }^{13} \mathrm{C}$ chemical shifts were referenced to TMS, whereas the ${ }^{113} \mathrm{Cd}$ ones were referenced respect to the signal of the secondary reference $\mathrm{Cd}\left(\mathrm{NO}_{3}\right)_{2} \cdot 4 \mathrm{H}_{2} \mathrm{O}\left(\delta{ }^{113} \mathrm{Cd}\right.$ of -100 ppm against the primary reference $0.1 \mathrm{M}$ aqueous solution of $\left.\mathrm{Cd}\left(\mathrm{ClO}_{4}\right)_{2}\right)$.

\section{RESULTS}

\subsection{Structural characterization by powder XRD}

In order to reach the objective of preparing Cd-based MOF-74-like materials, we systematically studied different $\mathrm{Cd}$ sources and different reported methods of synthesis of MOF$74 / \mathrm{CPO}-27-\mathrm{M} / \mathrm{M}_{2}(\mathrm{dhtp}) / \mathrm{M}_{2}$ (dobdc) materials. However, the aim of this article is not to report and discuss the failed conditions that do not lead to pure Cd-MOF-74. Nevertheless, it is pertinent to pointed out that the key point to obtain high-quality Cd-MOF-74 materials seemed to be the use of $\mathrm{Cd}$ acetate as $\mathrm{Cd}$ source. The use of nitrate source, $\mathrm{Cd}\left(\mathrm{NO}_{3}\right)_{2}$, which is the most commonly-used anion source for any M-MOF-74 / CPO-27 materials, systematically led to samples with impurities and/or poor in the desirable phase MOF-74 compared to its analogous samples prepared with $\mathrm{Cd}$ acetate. Some examples of the convenience of using acetate versus nitrate as Cd source are given in Supporting Information.

Figure 1 shows the powder X-ray diffraction (PXRD) patterns of the Cd-MOF-74 sample and a Zn-MOF-74 sample prepared according to the literature[13]. Similarities between both patterns are evident, suggesting that the MOF crystallized from our Cd-dhtp system probably has the topology of MOF-74 / CPO-27 materials (three-letter code of this topology is etb). At the same 
time, some differences between both XRD patterns are also patent. Apart from the presence/absence of some reflections, the most evident difference is the significant $2 \Theta$ shifts of the a priori equivalent reflections. These shifts must be due to the larger cation size of $\mathrm{Cd}^{2+}$ with respect that of $\mathrm{Zn}^{2+}$ (109 and $88 \mathrm{pm}$, respectively, for octahedral coordination). The high magnitude of the shifts, as large as $0.59^{\circ}$ for the reflection 300 , discards that its origin is only due to experimental error, particularly considering that metal Si has been used as internal reference of $2 \theta$ positions and that $2 \Theta$ shifts are not systematic (for instance, it is of $0.37^{\circ}$ for the reflection 110).

The structural resolution based on single-crystal X-ray methods was not possible as enough large high-quality crystals could not be achieved. However, the experimental PXRD pattern shown in Figure 1 allowed us estimating the unit cell parameters assuming that the trigonal spacetial group is-R-3 (no. 148), which is the one corresponding to the Zn-MOF-74 material[13]. The results of such estimation for the experimental patterns of the Zn-MOF-74 and Cd-MOF-74 samples are shown in Table 1. Although the goodness of the method could be somehow questionable attending to the deviation estimated for the unit cell parameter $a(0.33 \AA)$ of the sample Zn-MOF-74 with respect to that reported for that sample[13], the magnitude of that deviation is significantly smaller $(0.08$ and $0.07 \AA)$ when compared with the most recent unit cell parameters reported[15, 33] for the same Zn-MOF-74 materials and using the same space group (see footnote of the Table 1). In any case, the increase of unit cell parameters $a$ and $b$ from $\mathrm{Zn}$ - to Cd-MOF-74 material is out of any doubt as it is at least $1 \AA$ larger in the case of the Cd-MOF-74 materials. On the contrary, parameter $c$ is practically unaffected by the substitution of $\mathrm{Zn}$ by $\mathrm{Cd}$. The increase of $a$ and $b$ values must be related to the larger size of $\mathrm{Cd}^{2+}$, which will presumably lead to a slightly bigger micropore diameter. Although a significant increase in $c$ dimension was 
also expected, the either low or negligible sensitivity of $c$ to the size of metal ion in isomorphic substitution in other microporous materials with 1-D pore dimensionality and hexagonal space group, has been also found in the literature[34, 35] and, more significant, similar behavior has been found between $\mathrm{Zn}$ - and Cd-based MOFs with trigonal space group [28, 31].

The XRD-based analysis presented so far strongly suggests that our sample is a Cd-MOF-74 material. However, it cannot be taken as an absolute guarantee, particularly considering the evident differences between both XRD patterns of Figure 1 (different relative intensity of the peaks, absence/presence of certain reflections, etc.). Figure 2 compares the XRD patterns shown in Figure 1 as well as these of a Zn-MOF-74 sample prepared according to the literature[13] and a theoretical Cd-MOF-74. Since Cd-MOF-74 has not been described, its theoretical pattern has been generated starting from the CIF file of Zn-MOF-74, in which Zn has been substituted by $\mathrm{Cd}$, and taking the unit cell parameters estimated four our sample (Table 1). The agreement between both experimental and theoretical XRD patterns of Cd-MOF-74 is quite good. It is even better than that between experimental and theoretical patterns of Zn-MOF-74. Therefore,-any difference between the XRD patterns of Zn- and Cd-MOF-74 can be justified and attributed to the differences originated by the substitution of $\mathrm{Zn}^{2+}$ by $\mathrm{Cd}^{2+}$ ions. A couple of Figures showing the Cd-MOF-74 structure taken for simulating the theoretical PXRD patterns of Figure 2, are presented in Supplementary Information.

\subsection{IR spectroscopy}

Like powder X-ray diffraction patterns, IR spectroscopy region of $c a \cdot 650-1800 \mathrm{~cm}^{-1}$ is commonly taken as fingerprint of materials, especially when they contain organic entities. Whereas PXRD is particularly sensitive to the structure of materials (long-range information), 
that region of infrared spectroscopy is more sensitive to the conformational and/or local environment of organic molecules (short-range information), so both techniques are somehow complementary. Figure 3 shows the ATR-FTIR spectra of the Cd-MOF-74 and Zn-MOF-74 materials, and that of the free protonated linker dhtp. Similarities between the IR spectra of both M-MOF-74 are manifest, and then it can be assumed that both follow a common pattern. In contrast, the spectrum of the linker dhtp presents more differences than similarities with such pattern. Therefore, the here-described Cd-MOF-74 material has not only a similar crystalline ordering to the Zn-MOF-74 material (Figure 1), but also the organic linker environment is practically equal (Figure 3), strongly indicating that it is indeed a Cd-based MOF-74 / CPO-27 material.

\subsection{Themogravimetric analyses}

One of the most critical features of MOF materials is their thermal stability, which determines some of their potential applications. The thermal stability of M-MOF-74 materials toughly depends on the divalent metal nature[36]. Under air flow, Co-, Ni- and Mn-based materials decompose at lower temperatures than the free protonated linker dhtp, whereas $\mathrm{Zn}$ - and especially Mg-MOF-74 materials are quite more stable.

Figure 4 shows the TGA plot of the Cd-MOF-74 sample compared with these of a conventional Zn-MOF-74 material and the linker dhtp. Taking the linker decomposition temperature as an evaluation of the thermal stability, Cd-MOF-74 sample has practically the same stability than the linker and it is more or less in the middle of the stability scale formed by the known M-MOF-74 [30]. 
Table 2 shows some data extracted from the TGA plots of Figure 4. TGA profile of Cd-MOF74 is different to that of the Zn-MOF-74 not only in terms of the already-mentioned linker decomposition temperature but also in the amount of residue, i.e. in the amount of remaining weight once all organic part has been removed. The remaining material after TGA measurements of $\mathrm{Cd}$ - and $\mathrm{Zn}-\mathrm{MOF}-74$ samples were identified as $\mathrm{CdO}$ and $\mathrm{ZnO}$, respectively, by $\mathrm{XRD}$ characterization (patterns not shown). The identification as $\mathrm{ZnO}$ is in good agreement with the phase found after a similar TG analysis of a Zn-MOF-74 sample reported elsewhere[36]. Similarly, it was also expected that $\mathrm{CdO}$ was formed after the TG experiment of the sample $\mathrm{Cd}$ MOF-74 under air flow up to $900{ }^{\circ} \mathrm{C}$. The difference in the amount of the residual weight between $\mathrm{Cd}$ (48 wt. \%) and Zn (34 wt. \%) samples must be related to the heavier atomic weight of Cd compared to that of the $\mathrm{Zn}$. Accordingly, the linker-to-residue ratios of both samples, which are quite different if expressed in wt. \% / wt. \% (penultimate column of Table 2), become practically equal if they are corrected by the molecular weight of the corresponding residual MO species (last column of Table 2).

\section{4. $\mathrm{N}_{2}$ adsorption/desorption isotherm}

Figure 5 shows the $\mathrm{N}_{2}$ adsorption/desorption isotherms of a conventional Zn-MOF-74 and the Cd-MOF-74 material. Some relevant data from the isotherms of Figure 5 are compiled in Table 3. Cd-MOF-74 possesses high surface area but significantly lower than that of Zn-MOF-74. That difference must be again related to the heavier nature of $\mathrm{Cd}$ with respect to $\mathrm{Zn}[8]$. In this $\mathrm{MOF}$ material, the difference in atomic weight of metal is particularly important due to their high metal concentration (molecular formula $\mathrm{M}_{2}(\mathrm{dhtp})$ ). Indeed, the molecular weight of $\mathrm{Cd}_{2}(\mathrm{dhtp})$ is ca. 1.3 times that of $\mathrm{Zn}_{2}(\mathrm{dhtp})$, so the molar surface areas of both samples are quite similar, even that of Cd-MOF-74 is slightly higher (last column of Table 3). Another distinguished feature of 
Figure 5 is the presence of some adsorption in the mesopore range in the isotherm of Cd-MOF74 , evidenced by both the amount of adsorbed $\mathrm{N}_{2}$ in the $\mathrm{p} / \mathrm{p}_{0}$ range of $0.1-0.95$ as well as the existence of a hysteresis loop. Accordingly, the application of $t$-plot method indicates certain external surface area in this sample $\left(69 \mathrm{~m}^{2} \mathrm{~g}^{-1}\right)$. Maybe that extra contribution is the key point making the sample Cd-MOF-74 of higher molar surface area than its homologous Zn-MOF-74 sample (right column of Table 3).

\subsection{Scanning Electronic Microscopy (SEM)}

Figure 6 shows two SEM images of the Cd-MOF-74 material at different magnifications. The crystals have rod-like shape, typical of MOF-74 crystals prepared with other metal ions[15]. These crystals are agglomerated in elongated particles with a length of several micrometers. The agglomeration of the crystals could explain the amount of N2 adsorbed in the mesopore region in the isotherm of Cd-MOF-74 (Figure 5), as well as the presence of the hysteresis loop. Particles or crystals with different morphology could not be identified at any magnification, so the images of Figure 6 can be considered as representative of the all particles forming sample, which is quite homogeneous.

\subsection{Multinuclear NMR}

The diamagnetic character of Cd should make easier any NMR-based study of the MOF-74 compared to that of analogous M-MOF-74 materials with $\mathrm{M}$ being a paramagnetic divalent metal ion such as $\mathrm{Co}^{2+}, \mathrm{Cu}^{2+}, \mathrm{Fe}^{2+}, \mathrm{Ni}^{2+}$ or $\mathrm{Mn}^{2+}$. Unlike them, $\mathrm{Zn}^{2+}$ and $\mathrm{Mg}^{2+}$, the known diamagnetic divalent metal ions able to lead the corresponding M-MOF-74 materials, do not avoid the study of other neighbor nuclei of the system such as ${ }^{1} \mathrm{H}$ or ${ }^{13} \mathrm{C}[37]$. However, their own 
NMR parameters are quite unfavorable in terms of NMR-sensitivity for high-resolution spectra, making hard and time-consuming any NMR study[32] on the active role of the open metal sites of these materials in catalytic or adsorption processes. In contrast, NMR sensitivity of ${ }^{111} \mathrm{Cd}$ or ${ }^{113} \mathrm{Cd}$ guarantees reasonable good NMR response in any process involving unsaturated and/or exposed Cd sites. Figures 7 and 8 are two good examples of both NMR features introduced by the discover of Cd-MOF-74. Figure 7 shows ${ }^{13} \mathrm{C}$ NMR spectra of Cd- and Zn-MOF-74 materials whose high resolution is not viable for M-MOF-74 materials having a paramagnetic M. Figure 8 shows the first high resolution NMR spectra of a diamagnetic non-quadrupolar open metal nucleus $\left({ }^{113} \mathrm{Cd}\right)$ of a M-MOF-74 material.

To gain signal-to-noise ratio, ${ }^{13} \mathrm{C}$ MAS NMR spectra shown in Figure 7 were registered by applying ${ }^{1} \mathrm{H}$ to ${ }^{13} \mathrm{C}$ cross polarization technique and under ${ }^{1} \mathrm{H}$ decoupling. ${ }^{13} \mathrm{C}$ chemical shifts $(\delta$ ${ }^{13} \mathrm{C}$ ) were the same either by cross polarization or by direct ${ }^{13} \mathrm{C}$ radiation (spectra not shown). Therefore, the systematic modifications of $\delta{ }^{13} \mathrm{C}$ clearly observed in every ${ }^{13} \mathrm{C}$ signal between the spectra of any M-MOF-74 and the protonated organic linker dhtp, should not be assigned to the NMR pulse sequence but exclusively to real differences in chemical environment. The downfield shifts of $\delta{ }^{13} \mathrm{C}$ could be interpreted by a greater electron donor character of the divalent metals, $\mathrm{Zn}$ and $\mathrm{Cd}$, with respect to $\mathrm{H}$ atoms, as expected from the higher iconicity of the formers. The same interpretation can be attributed to the slight downfield shifts of $\delta{ }^{13} \mathrm{C}$ of the Cd-MOF-74 spectrum with respect to these of its homologous Zn-MOF-74, as the ionic character of $\mathrm{Cd}$ is greater. Accordingly, the highest shift is detected in the $\delta{ }^{13} \mathrm{C}$ of position d (Figure 7), i.e. in the $\mathrm{C}$ atom of the carboxylate group directly linked to $\mathrm{Cd}$ (or $\mathrm{Zn}$ ). The better signal-to-noise of the Zn-MOF-74 spectrum as well as their narrower ${ }^{13} \mathrm{C}$ signals must be due to a higher crystal size as 
well as to the negligible ${ }^{67} \mathrm{Zn}-{ }^{13} \mathrm{C}$ couplings compared to some possible non-negligible ${ }^{113} \mathrm{Cd}-{ }^{13} \mathrm{C}$ and ${ }^{111} \mathrm{Cd}-{ }^{13} \mathrm{C}$ magnetic interactions, which could widen the ${ }^{13} \mathrm{C}$ signal at some extension.

Figure 8 shows the single-pulse ${ }^{113} \mathrm{Cd}$ MAS NMR spectrum and ${ }^{1} \mathrm{H}$ to ${ }^{113} \mathrm{Cd} \mathrm{CP}$ MAS NMR spectrum of the Cd-MOF-74 sample. Both spectra are dominated by a signal at $c a .40 \mathrm{ppm}$, which is attributed to the $\mathrm{Cd}$ in the only crystallographic position in the MOF-74 structure. That assignment is supported by the relative proximity of $\delta{ }^{113} \mathrm{Cd}$ to these found in the spectra of similar octahedral environments surrounding by 6 oxygen atoms such as cadmium ammonium sulfate hexahydrate (61 ppm), cadmium formate dihydrate $(26,21 \mathrm{ppm})$, cadmium maleate dihydrate $(12,-7 \mathrm{ppm})$, cadmium perchlorate hexahydrate $(0 \mathrm{ppm}$, the most common primary reference for ${ }^{113} \mathrm{Cd}$ )[38]. (Cadmium acetate dihydrate has a $\delta{ }^{113} \mathrm{Cd}$ of $-46 \mathrm{ppm}[38]$ but $\mathrm{Cd}$ is actually surrounded by 7 oxygen atoms[39]). Although that chemical shift range from 61 to -7 ppm could seem too large, it must be taken into account that $\delta{ }^{113} \mathrm{Cd}$ range covers several hundreds of $\mathrm{ppm}[38]$. In other words, $\delta{ }^{113} \mathrm{Cd}$ is extraordinarily sensitive to minimum changes in Cd environment, which provides an extra advantage to the use of ${ }^{113} \mathrm{Cd}$ as a probe of reaction / adsorption / (de)solvation mechanisms involving the open metal sites in Cd-MOF-74 materials.

${ }^{113}$ Cd MAS NMR spectrum has a broad shoulder of the main signal. Such shoulder was not detected in the CP MAS NMR spectrum. It could be due to the presence of some Cd-containing impurities in the Cd-MOF-74 sample. These impurities could probably correspond to extraframework species with similar environment to that found in the Cd-MOF-74 (the difference of ca. $30 \mathrm{ppm}$ should not be considered as significant according to discussion in the previous paragraph) and of high mobility (since the quite abundant ${ }^{1} \mathrm{H}$ nuclei are not able to transfer the polarization to ${ }^{113} \mathrm{Cd}$ nuclei). 


\section{DISCUSSION}

Since the basic results have been already discussed, this section focuses on underlining the scope of this work. First of all, $\mathrm{Cd}^{2+}$ is presented as the first divalent metal ion of the second transition metal row able to form a MOF material with etb topology using dhtp as organic linker. In this sense, Cd-MOF-74 is not merely one MOF-74 material but $\mathrm{Cd}^{2+}$ ion is significantly larger than the divalent cations $\mathrm{M}$ of the known M-MOF-74 materials. Therefore, it is expected that $\mathrm{Cd}$ could generate larger pores for that given structure, which should be beneficial for gas storage and/or catalytic process involving relatively large molecules. Nevertheless, admitting that IRMOF-74 materials also belong to the family of MOF-74 materials, the expansion of pore size dimension given by Cd-MOF-74 is rather poor compared with that given by, for instance, IRMOF-74-XI[23]. Even though, the discover of Cd-MOF-74 would allow to outstandingly widen systematic studies on any physic-chemical property in the series of isostructural M-MOF74 materials, particularly on these studies in which $M$ nature plays a key role.

Moreover, one of the most cited articles of MOFs as heterogeneous catalysts is about a 2-D Cd-based MOF material[40], in which Cd ions are also exposed and unsaturated. Consequently, Cd-MOF-74 could potentially find promising catalytic properties, with the advantage of possessing $\mathrm{Cd}$ centers able to become unsaturated under controlled experimental conditions (vacuum and/or temperature). Furthermore, Cd-MOF-74 is a 3-D material and possesses welldefined micropores which could add shape-selectivity to the intrinsic catalytic activity of the $\mathrm{Cd}$ active centers. In addition, this structure has acceptable thermal and hydrothermal stability[16], which is an essential requirement for some catalytic applications. 
- Finally, the relatively good properties of two diamagnetic non-quadrupolar isotopes of Cd $\left({ }^{111} \mathrm{Cd}\right.$ and ${ }^{113} \mathrm{Cd}$ ) to be studied by NMR (both have $\mathrm{I}=1 / 2$; natural abundance of 12.75 and 12.26 $\%$, respectively; gyromagnetic ratio of 5.96 and $5.70 \cdot 10^{-7} \mathrm{radT}^{-1} \mathrm{~s}^{-1}$, respectively; chemical shift ranges from -850 to $2000 \mathrm{ppm}$ for both nuclei) offers new possibilities to study the interaction between the exposed metal sites and any adsorbate / reactant from the metal point of view. Such information could be crucial to understand both the mechanism and the role of the open metal sites in adsorption/catalytic processes. It should definitely provide a quicker and more direct information than that found in the meritorious publication focused on ${ }^{25} \mathrm{Mg}$ MAS NMR of $\mathrm{Mg}$ MOF-74[32] $\left({ }^{25} \mathrm{Mg}\right.$ nucleus has a spin I of 5/2 and a quadrupolar moment of $0.22 \times 10^{28} \mathrm{~m}^{2}$, natural abundance of $10.1 \%$, gyromagnetic ratio of $1.64 \cdot 10^{-7} \mathrm{radT}^{-1} \mathrm{~s}^{-1}$, chemical shift range of about $-20 / 50 \mathrm{ppm})$. Similarly, ${ }^{113} \mathrm{Cd}$ or ${ }^{111} \mathrm{Cd}$ NMR could provide insights and/or indications about some other significant features such as the presence/absence of $\mathrm{Cd}$ containing impurities (this work can take as an example), possible damage suffered by the Cd-MOF material after certain treatments, accessibility of a given reactant/ adsorbate to the metal centers, or exposure/solvation degree of the metal ions of the MOF-74 framework.

\section{CONCLUSSIONS}

The preparation of a Cd-based MOF-74-like material has been described. The use of $\mathrm{Cd}$ acetate as $\mathrm{Cd}$ source was found to be one of the most critical keys to afford pure crystalline phase. Nevertheless, no crystals of enough size and/or quality were obtained to allow us its structural resolution by single-crystal X-ray techniques. However, the MOF-74 nature of the Cd-based sample has been deduced by a combination of different characterization techniques. Because of the larger size of the Cd with respect that of the known metal able to lead M-MOF-74, the Cd- 
MOF-74 unit cell is larger in the plane $a-b$, and presumably their pore diameter is also larger. Powder XRD diffraction matches quite well with the theoretical XRD pattern generated by the substitution of $\mathrm{Zn}$ by $\mathrm{Cd}$ ions in a theoretical Zn-MOF-74 material. Its thermal stability under air conditions is lower than its homologues based on $\mathrm{Zn}$ and $\mathrm{Mg}$, but it is more stable than those materials prepared by $\mathrm{Co}, \mathrm{Ni}, \mathrm{Mn}$ or $\mathrm{Cu}$. Finally, the NMR characterization of the M-MOF-74 from a relative sensitive open metal site $\mathrm{M}$ isotope $\left({ }^{113} \mathrm{Cd}\right)$ is made clear and opens some exceptional possibilities for future studies involving interactions of the open metal sites with adsorbate molecules, reactants or solvents.

\section{ACKNOWLEDGMENTS}

We appreciate the effort done by Conchi Díaz and Rosa Folgado from 'ICP Unidad de Apoyo' in the registration of PXRD patterns and $\mathrm{N}_{2}$ isotherms, respectively. Spanish Ministries are acknowledged for financial support (MAT-2009-13569 and MAT-2012-31127).

\section{REFERENCES}

[1] Issue 2 Chem. Rev., 112 (2012) 673-1268.

[2] R.J. Kuppler, D.J. Timmons, Q.-R. Fang, J.-R. Li, T.A. Makal, M.D. Young, D. Yuan, D.

Zhao, W. Zhuang, H.-C. Zhou, Potential applications of metal-organic frameworks, Coordination Chemistry Reviews, 253 (2009) 3042-3066.

[3] B.L. Chen, M. Eddaoudi, S.T. Hyde, M. O'Keeffe, O.M. Yaghi, Interwoven metal-organic framework on a periodic minimal surface with extra-large pores, Science, 291 (2001) 1021-1023. [4] C. Serre, F. Millange, C. Thouvenot, M. Nogues, G. Marsolier, D. Louer, G. Ferey, Very large breathing effect in the first nanoporous chromium(III)-based solids : Mil-53 or $\mathrm{CrIII}(\mathrm{OH}) .\{\mathrm{O} 2 \mathrm{C}-\mathrm{C} 6 \mathrm{H} 4-\mathrm{CO} 2\} .\{\mathrm{HO} 2 \mathrm{C}-\mathrm{C} 6 \mathrm{H} 4-\mathrm{CO} 2 \mathrm{H}\}$ x.H2Oy, J. Am. Chem. Soc., 124 (2002) 13519-13526.

[5] J.S. Seo, D. Whang, H. Lee, S.I. Jun, J. Oh, Y.J. Jeon, K. Kim, A homochiral metal-organic porous material for enantioselective separation and catalysis, Nature, 404 (2000) 982-986. [6] M. Dinca, J.R. Long, Hydrogen storage in microporous metal-organic frameworks with exposed metal sites, Angew Chem Int Ed Engl, 47 (2008) 6766-6779. 
[7] P.D.C. Dietzel, R. Blom, H. Fjellvag, Base-induced formation of two magnesium metalorganic framework compounds with a bifunctional tetratopic ligand, European Journal of Inorganic Chemistry, (2008) 3624-3632.

[8] S.R. Caskey, A.G. Wong-Foy, a.A.J. Matzger, Dramatic Tuning of Carbon Dioxide Uptake via Metal Substitution in a Coordination Polymer with Cylindrical Pores, J. Am. Chem. Soc., 130 (2008) 10870.

[9] W. Zhou, H. Wu, T. Yildirim, Enhanced H2 Adsorption in Isostructural Metal-Organic Frameworks with Open Metal Sites: Strong Dependence of the Binding Strength on Metal Ions, J. Am. Chem. Soc., 130 (2008) 15268-15269.

[10] S. Bhattacharjee, J.S. Choi, S.T. Yang, S.B. Choi, J. Kim, W.S. Ahn, Solvothermal Synthesis of Fe-MOF-74 and Its Catalytic Properties in Phenol Hydroxylation, Journal of Nanoscience and Nanotechnology, 10 (2010) 135-141.

[11] P.D.C. Dietzel, Y. Morita, R. Blom, H. Fjellvag, An in situ high-temperature single-crystal investigation of a dehydrated metal-organic framework compound and field-induced magnetization of one-dimensional metaloxygen chains, Angew. Chem.-Int. Edit., 44 (2005) 6354-6358.

[12] P.D. Dietzel, B. Panella, M. Hirscher, R. Blom, H. Fjellvag, Hydrogen adsorption in a nickel based coordination polymer with open metal sites in the cylindrical cavities of the desolvated framework, Chemical Communications (Cambridge, United Kingdom), (2006) 959961.

[13] N.L. Rosi, J. Kim, M. Eddaoudi, B.L. Chen, M. O'Keeffe, O.M. Yaghi, Rod packings and metal-organic frameworks constructed from rod-shaped secondary building units, J. Am. Chem. Soc., 127 (2005) 1504-1518.

[14] P.D. Dietzel, R.E. Johnsen, R. Blom, H. Fjellvag, Structural changes and coordinatively unsaturated metal atoms on dehydration of honeycomb analogous microporous metal-organic frameworks, Chemistry, 14 (2008) 2389-2397.

[15] J.A. Botas, G. Calleja, M. Sanchez-Sanchez, M.G. Orcajo, Effect of Zn/Co ratio in MOF-74 type materials containing exposed metal sites on their hydrogen adsorption behaviour and on their band gap energy, Int. J. Hydrogen Energy, 36 (2011) 10834-10844.

[16] S.S. Han, S.H. Choi, A.C. van Duin, Molecular dynamics simulations of stability of metalorganic frameworks against $\mathrm{H} 2 \mathrm{O}$ using the ReaxFF reactive force field, Chem Commun (Camb), 46 (2010) 5713-5715.

[17] D.J. Tranchemontagne, J.R. Hunt, O.M. Yaghi, Room temperature synthesis of metalorganic frameworks: MOF-5, MOF-74, MOF-177, MOF-199, and IRMOF-0, Tetrahedron, 64 (2008) 8553-8557.

[18] M.P. Suh, H.J. Park, T.K. Prasad, D.W. Lim, Hydrogen storage in metal-organic frameworks, Chemical reviews, 112 (2012) 782-835.

[19] K. Sumida, D.L. Rogow, J.A. Mason, T.M. McDonald, E.D. Bloch, Z.R. Herm, T.H. Bae, J.R. Long, Carbon dioxide capture in metal-organic frameworks, Chemical reviews, 112 (2012) 724-781.

[20] X.F. Wu, Z.B. Bao, B. Yuan, J. Wang, Y.Q. Sun, H.M. Luo, S.G. Deng, Microwave synthesis and characterization of MOF-74 $(\mathrm{M}=\mathrm{Ni}, \mathrm{Mg})$ for gas separation, Microporous and Mesoporous Materials, 180 (2013) 114-122.

[21] R. Sanz, F. Martinez, G. Orcajo, L. Wojtas, D. Briones, Synthesis of a honeycomb-like Cubased metal-organic framework and its carbon dioxide adsorption behaviour, DTr, 42 (2013) 2392-2398. 
[22] L. Sun, T. Miyakai, S. Seki, M. Dinca, Mn(2,5-disulfhydrylbenzene-1,4-dicarboxylate): A Microporous Metal-Organic Framework with Infinite (-Mn-S-) Chains and High Intrinsic Charge Mobility, J. Am. Chem. Soc., (2013).

[23] H. Deng, S. Grunder, K.E. Cordova, C. Valente, H. Furukawa, M. Hmadeh, F. Gandara, A.C. Whalley, Z. Liu, S. Asahina, H. Kazumori, M. O'Keeffe, O. Terasaki, J.F. Stoddart, O.M. Yaghi, Large-pore apertures in a series of metal-organic frameworks, Science, 336 (2012) 10181023.

[24] D.F. Sun, Y.X. Ke, D.J. Collins, G.A. Lorigan, H.C. Zhou, Construction of robust open metal-organic frameworks with chiral channels and permanent porosity, Inorg. Chem., 46 (2007) 2725-2734.

[25] W.Q. Kan, J. Yang, Y.Y. Liu, J.F. Ma, A series of metal-organic frameworks based on 9,10bis(imidazol-1-ylmethyl) anthracene and structurally related aromatic dicarboxylates: Syntheses, structures, and photoluminescence, Polyhedron, 30 (2011) 2106-2113.

[26] Z. Zhang, K. Hu, Y.J. Shi, J.M. Qin, Z.L. Chen, F.P. Liang, Hydrothermal syntheses, crystal structures and fluorescent properties of five transition metal-organic hybrids incorporating an unsymmetrical benzotriazole carboxylate ligand, Transition Met. Chem., 38 (2013) 327-334. [27] L. Carlucci, G. Ciani, S. Maggini, D.M. Proserpio, M. Visconti, Heterometallic modular metal-organic 3D frameworks assembled via new tris-beta-diketonate metalloligands:

nanoporous materials for anion exchange and scaffolding of selected anionic guests, Chemistry, 16 (2010) 12328-12341.

[28] B.F. Abrahams, R.W. Elliott, T.A. Hudson, R. Robson, A new type of 3D

[(MII)2(TCNQ-II)3]2- coordination network with spacious channels of hexagonal cross-section generated from TCNQH2, CrystEngComm, 14 (2012) 351.

[29] J.-Y. Zhang, A.-L. Cheng, Q. Sun, Q. Yue, E.-Q. Gao, Syntheses, Structures, and Properties of Honeycomb and Square Grid Coordination Polymers with In Situ Formed 5-(2'-

Pyrimidyl)tetrazolate, Crystal Growth \& Design, 10 (2010) 2908-2915.

[30] D. Sun, Y. Ke, D.J. Collins, G.A. Lorigan, Z. Hong-Cai, Construction of Robust Open

Metal-Organic Frameworks with Chiral Channels and Permanent Porosity, Inorganic Chemistry, Vol. 46, No. 7, 2007 2725, 46 (2007) 2725-2734.

[31] T.-T. Luo, Y.-H. Liu, H.-L. Tsai, C.-C. Su, C.-H. Ueng, K.-L. Lu, A Novel Hybrid Supramolecular Network Assembled from Perfect ??? Stacking of an Anionic Inorganic Layer and a Cationic Hydronium-Ion-Mediated Organic Layer, European Journal of Inorganic Chemistry, 2004 (2004) 4253-4258.

[32] J. Xu, V.V. Terskikh, Y. Huang, 25Mg Solid-State NMR: A Sensitive Probe of Adsorbing Guest Molecules on a Metal Center in Metal-Organic Framework CPO-27-Mg, The Journal of Physical Chemistry Letters, 4 (2013) 7-11.

[33] W. Wong-Ng, J.A. Kaduk, H. Wu, M. Suchomel, Synchrotron X-ray studies of metalorganic framework M 2(2,5-dihydroxyterephthalate), $\mathrm{M}=(\mathrm{Mn}, \mathrm{Co}, \mathrm{Ni}, \mathrm{Zn})$ (MOF74), Powder Diffr., 27 (2012) 256-262.

[34] P. Concepcion, J.M. Lopez-Nieto, A. Mifsud, J. Perez-Pariente, Preparation and characterization of Mg-containing AFI and chabazite-type materials, Zeolites, 16 (1996) 56-64. [35] M. Sanchez-Sanchez, R. van Grieken, D.P. Serrano, J.A. Melero, On the Sn(II) and Sn(IV) incorporation into the AFI-structured AIPO4-based framework: the first significantly acidic SnAPO-5, Journal of Materials Chemistry, 19 (2009) 6833-6841. 
[36] S.A. FitzGerald, B. Burkholder, M. Friedman, J.B. Hopkins, C.J. Pierce, J.M. Schloss, B. Thompson, J.L. Rowsell, Metal-specific interactions of H2 adsorbed within isostructural metalorganic frameworks, J. Am. Chem. Soc., 133 (2011) 20310-20318.

[37] X. Kong, E. Scott, W. Ding, J.A. Mason, J.R. Long, J.A. Reimer, CO2 dynamics in a metalorganic framework with open metal sites, J. Am. Chem. Soc., 134 (2012) 14341-14344.

[38] P.G. Mennitt, M.P. Shatlock, V.J. Bartuska, G.E. Maciel, Cd-113 NMR-Studies of solid cadmium(II) complexes, J. Phys. Chem., 85 (1981) 2087-2091.

[39] W. Harrison, J. Trotter, Crystal and molecular structure of cadmium diacetate dihydrate, Journal of the Chemical Society-Dalton Transactions, (1972) 956-\&.

[40] M. Fujita, Y.J. Kwon, S. Washizu, K. Ogura, Preparataion, clathration ability, and catalysis of 2-dimensional square network material composed of cadmium(II) and 4,4' bipyridine, J. Am. Chem. Soc., 116 (1994) 1151-1152. 


\section{FIGURE CAPTIONS}

Figure 1. PXRD patterns of the $\mathrm{Cd}$ and $\mathrm{Zn}-\mathrm{MOF}-74$ materials. Miller indexes of the most intense reflections are labelled in both patterns. The difference in $2 \Theta$ values of the reflections 110 and 300 between both patterns is indicated.

Figure 2. Experimental (top) and theoretical (bottom) PXRD patterns of Cd-MOF-74 (black bold lines) and Zn-MOF-74 (red thin lines). Theoretical pattern of Zn-MOF-74 was generated from X-ray single crystal data[13] whereas that of Cd-MOF-74 was generated as explained in experimental part.

Figure 3. FTIR-ATR spectra of the free protonated linker dhtp (top), the Zn-MOF-74 sample conventionally prepared[13] (middle) and the Cd-MOF-74 material (bottom).

Figure 4. TGA plots of the Cd-MOF-74, a conventional Zn-MOF-74 material and the organic linker dhtp.

Figure 5. $\mathrm{N}_{2}$ adsorption/desorption isotherms at $-196^{\circ} \mathrm{C}$ of the $\mathrm{Cd}-\mathrm{MOF}-74$ material and a conventional Zn-MOF-74.

Figure 6. SEM micrographs of the Cd-MOF-74 sample at different magnifications: A) 16000; and B) 60000 . 
Figure 7. $100-200 \mathrm{ppm}$ region of the normalized ${ }^{1} \mathrm{H}$ to ${ }^{13} \mathrm{C}$ cross-polarization MAS NMR spectra of the Cd-MOF-74 (top) and a Zn-MOF-74 material (bottom). ${ }^{13} \mathrm{C}$ chemical shifts of the different $\mathrm{C}$ environments of the protonated organic linker dhtp in DMSO-d6 are indicated as dashed lines and identified by letters a, b, c and d, which correspondto the positions indicated over the protonated dhtp molecule drawing on the top-left corner.

Figure 8. Single-pulse ${ }^{113} \mathrm{Cd}$ (bottom) and ${ }^{1} \mathrm{H}$ to ${ }^{113} \mathrm{Cd}$ cross-polarization (top) MAS NMR spectra of the Cd-MOF-74 material. The position of the main peak of the CP NMR spectra is indicated. Asterisks indicate spinning-side bands in both spectra. 


\section{TABLES}

Table 1. Unit cell dimensions of the Cd-MOF-74 sample and a Zn-MOF-74 prepared according to a conventional method[13]. The theoretical values of the Zn-MOF-74 from the X-ray single crystal structure solving data are also included.

Table 2. TGA data extracted from the plots shown in Figure 4.

Table 3. Textural properties extracted from the plots shown in Figure 5. 


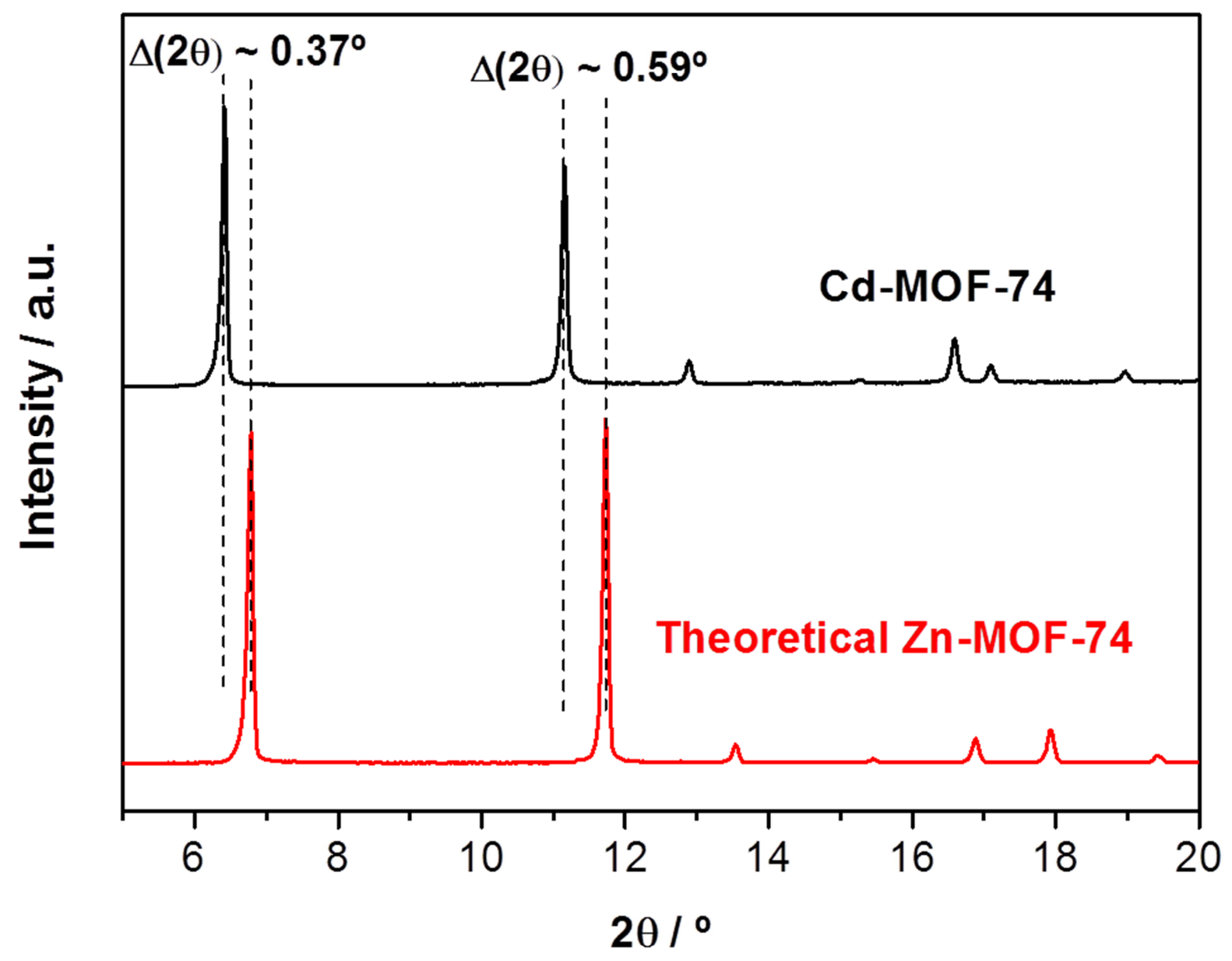

Figure 1 


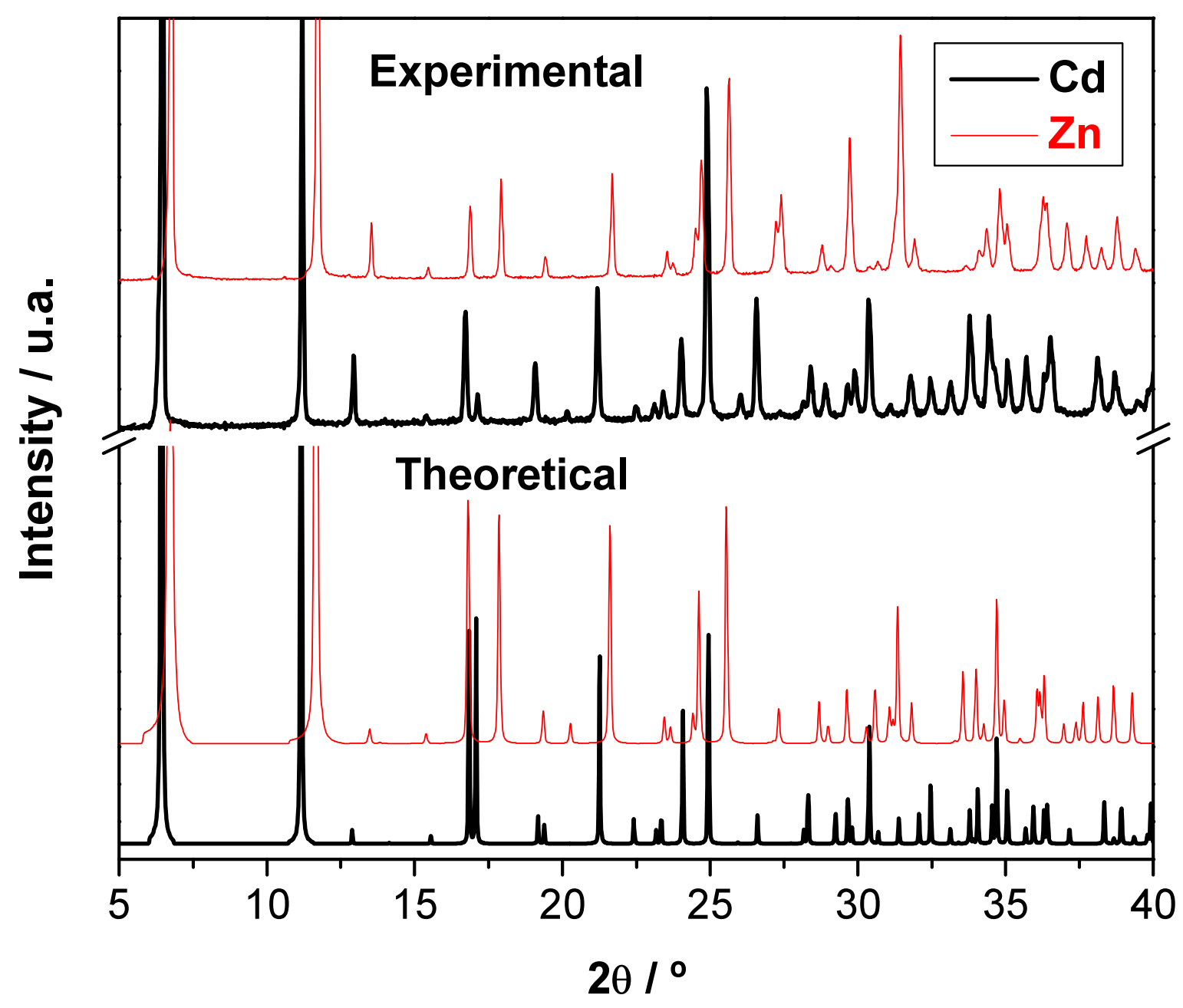

Figure 2 


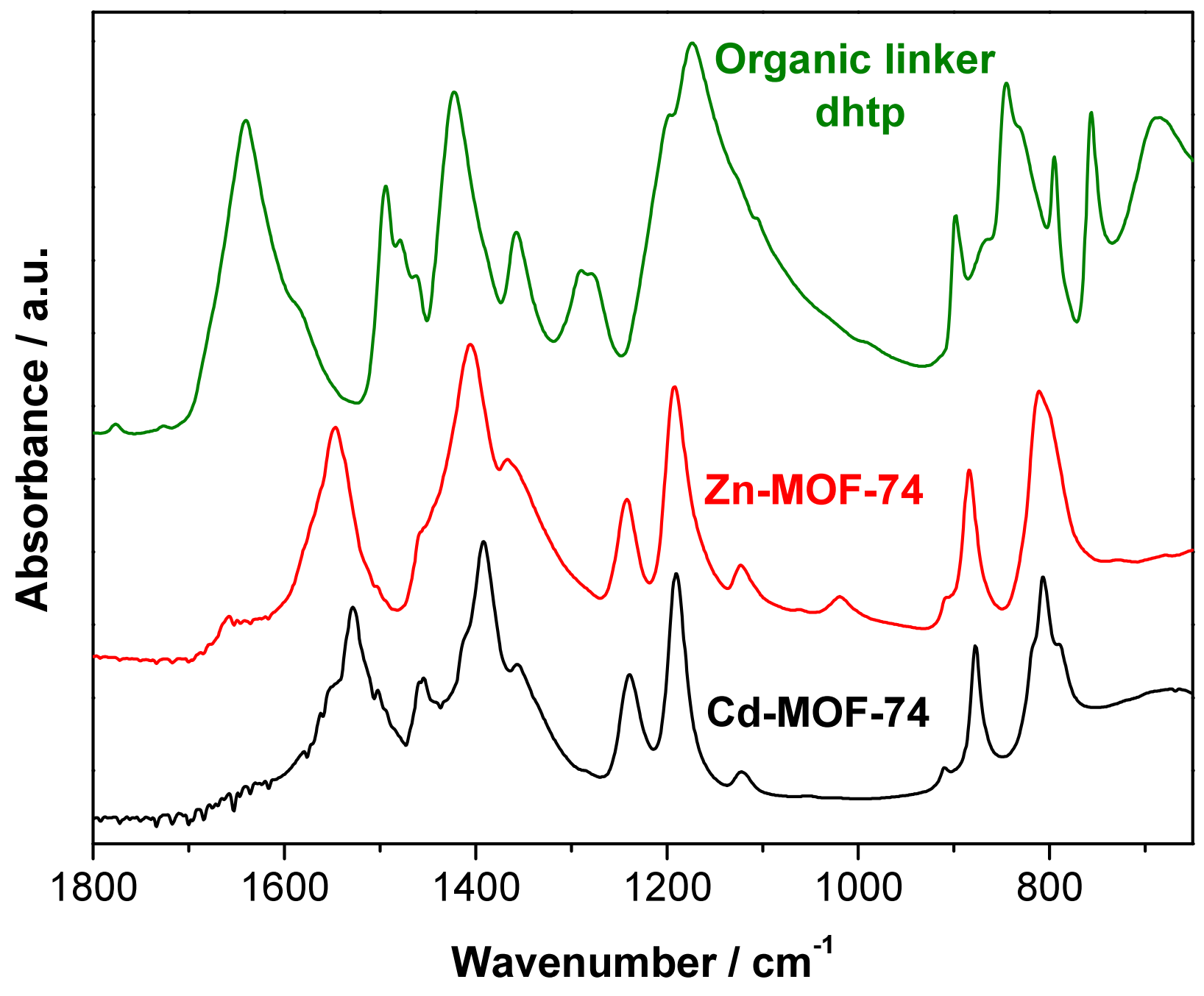

Figure 3 




Figure 4 


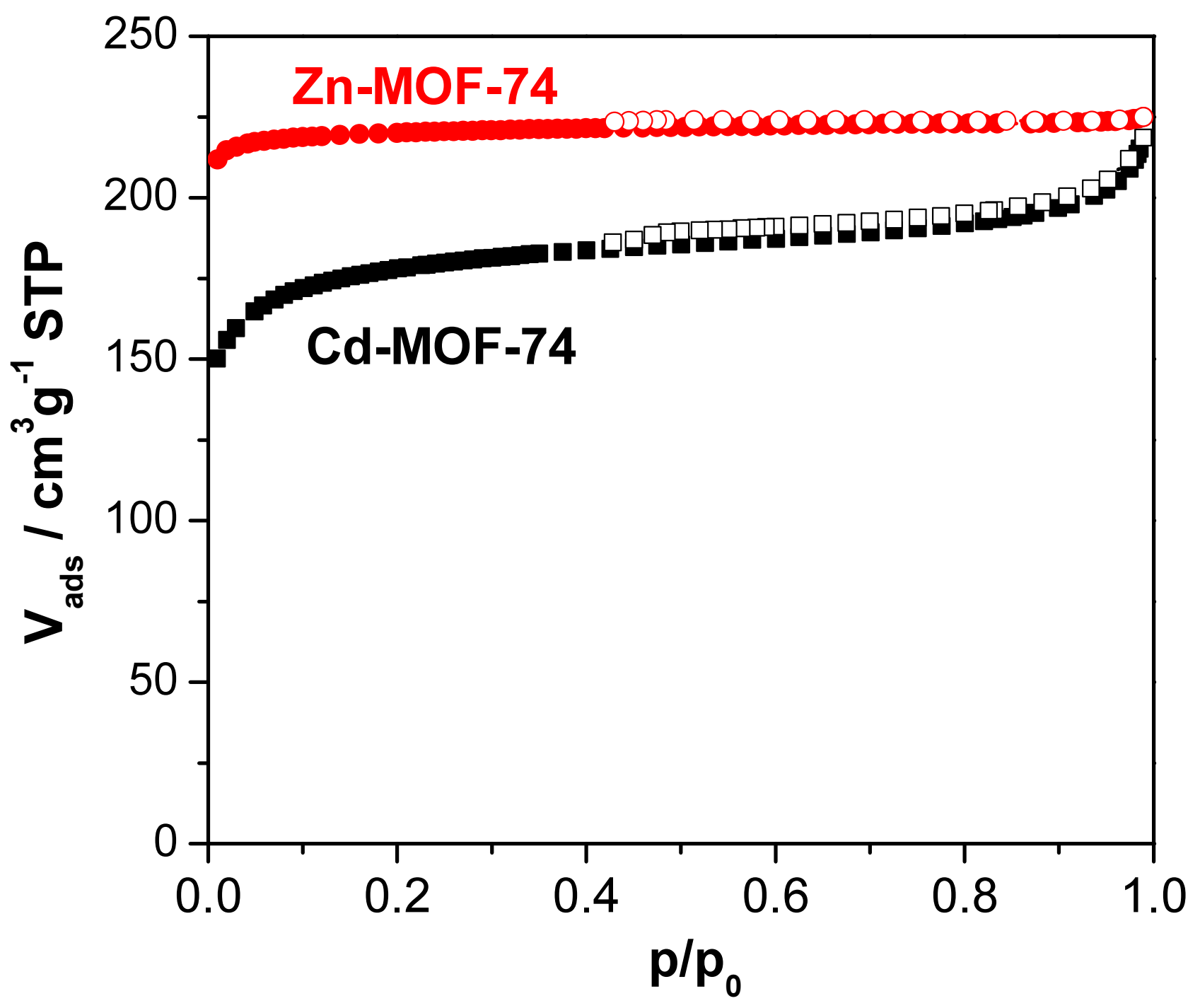

Figure 5 

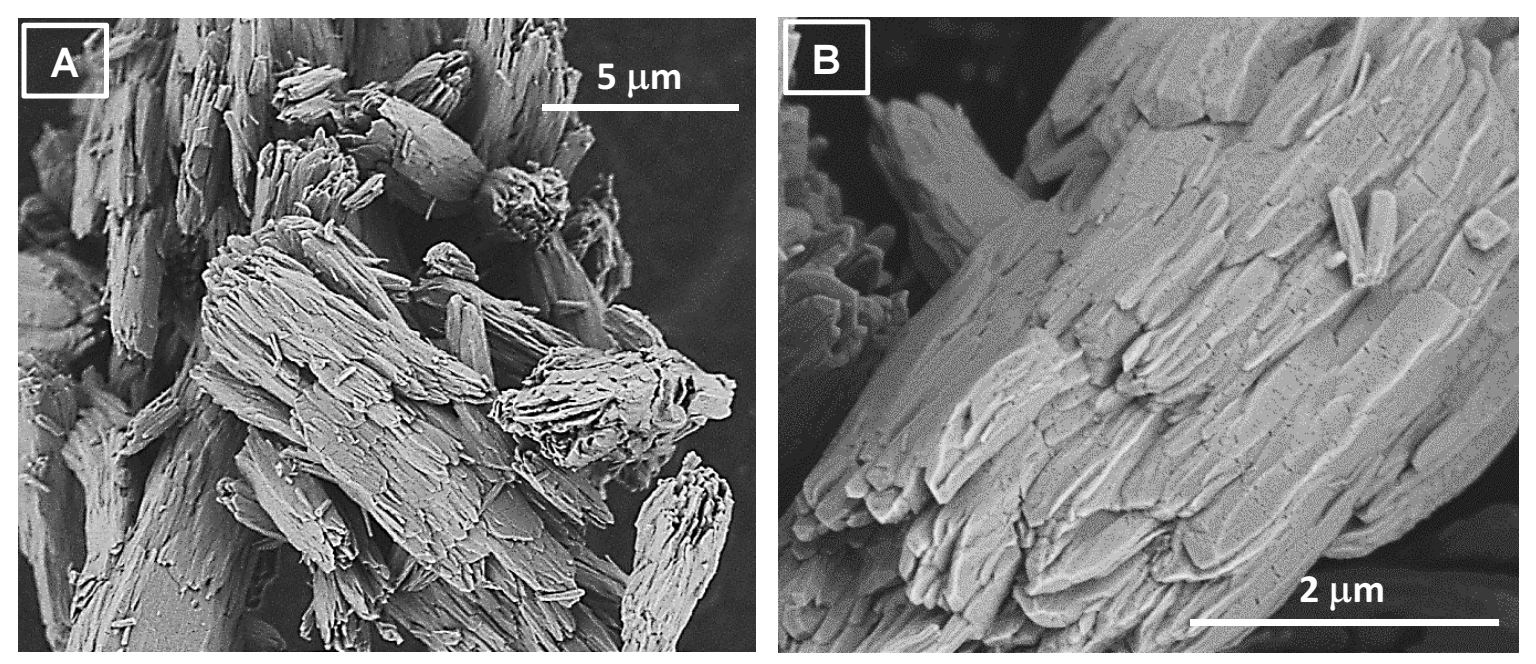

Figure 6 


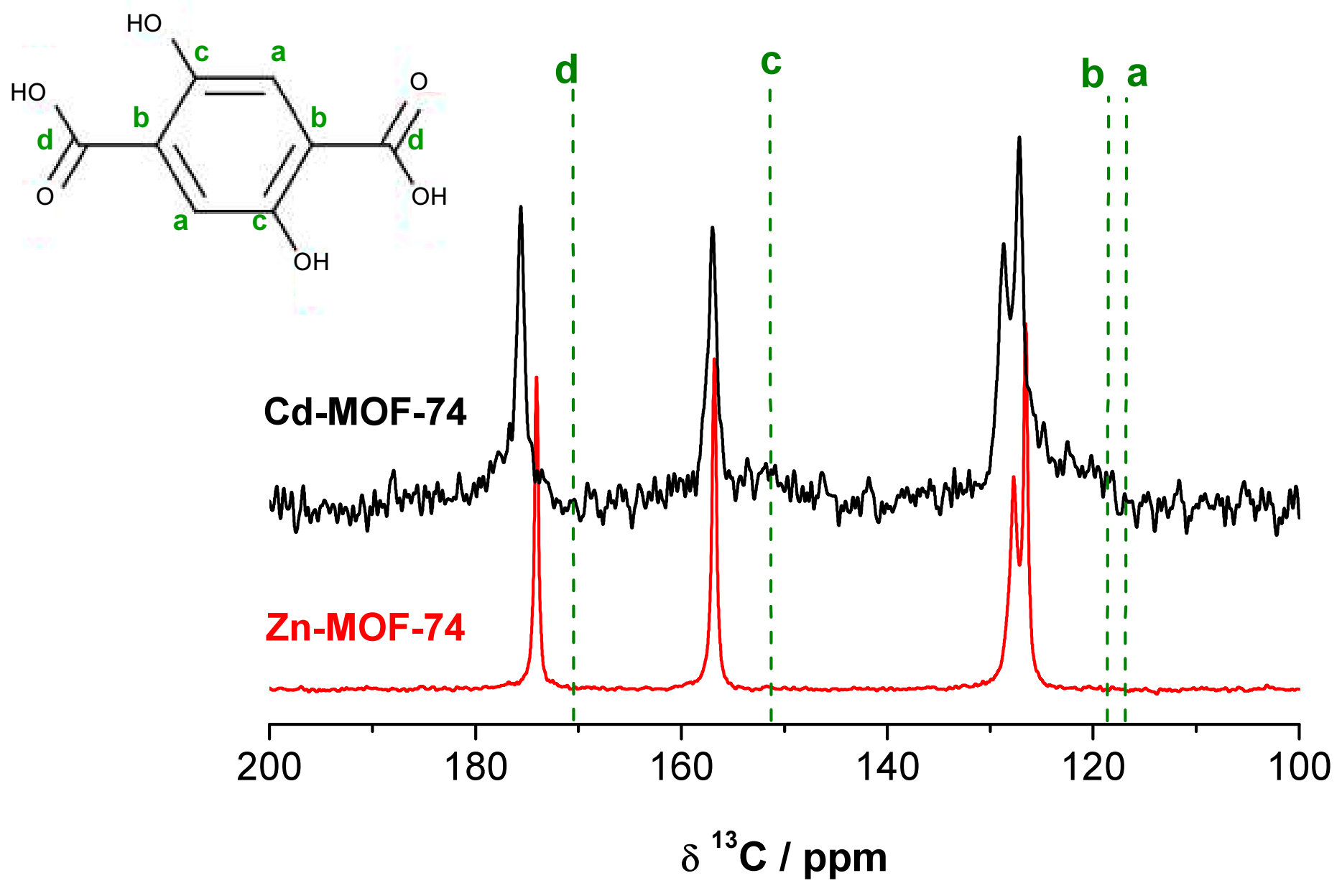

Figure 7 


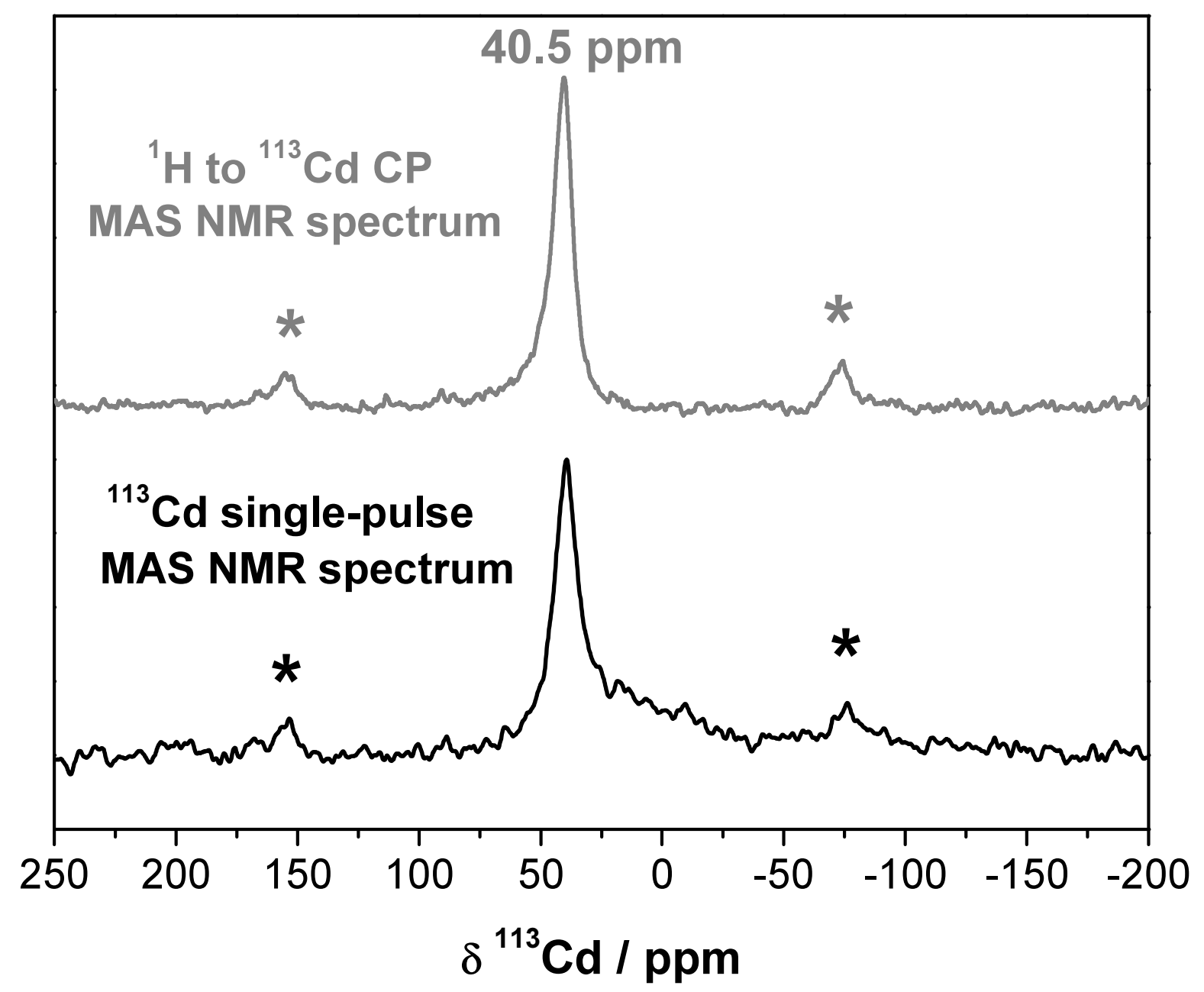

Figure 8 
Table 1. Unit cell dimensions of the Cd-MOF-74 sample and a Zn-MOF-74 prepared according to a conventional method[13]. The theoretical values of the Zn-MOF-74 from the X-ray single crystal structure solving data are also included.

\begin{tabular}{|c|c|c|}
\hline & \multicolumn{2}{|c|}{ Unit cell dimensions / $\AA$} \\
\hline M-MOF-74 material & $\mathrm{a}=\mathrm{b}$ & $\mathrm{c}$ \\
\hline Theoretical Zn-MOF-74 & $25.93^{\mathrm{a}}$ & $6.84^{\mathrm{a}}$ \\
\hline Zn-MOF-74 & 26.262 & 6.670 \\
\hline Cd-MOF-74 & 27.463 & 6.652 \\
\hline
\end{tabular}

${ }^{a}$ The most recent unit cell parameters reported in the literature for Zn-MOF-74 materials are of $26.18[33]$ and 26.19[15] for parameters $a$ and $b$, and 6.65[33] for parameter $c$. 
Table 2. TGA data extracted from the plots shown in Figure 4.

\begin{tabular}{|c|c|c|c|c|c|c|c|}
\hline \multirow[t]{2}{*}{ Sample } & \multicolumn{2}{|c|}{ Loss 1 (Solvent) } & \multicolumn{2}{|c|}{ Loss 2 (Linker) } & \multirow{2}{*}{$\begin{array}{c}\text { Residue } \\
\text { wt. \% }\end{array}$} & \multirow{2}{*}{$\begin{array}{c}\text { Loss } 2 / \text { residue } \\
\text { ratio }\end{array}$} & \multirow{2}{*}{$\begin{array}{l}\text { Loss } 2 / \mathrm{mol} \mathrm{MO}^{-1} \\
\text { ratio }\left(\mathrm{g} \cdot \mathrm{mol}^{-1}\right)^{\mathrm{a}}\end{array}$} \\
\hline & $\mathrm{T} /{ }^{\circ} \mathrm{C}$ & wt. \% & $\mathrm{T} /{ }^{\circ} \mathrm{C}$ & wt. \% & & & \\
\hline Zn-MOF-74 & 108 & 25.9 & 408 & 39.7 & 34.4 & 1.15 & 93.6 \\
\hline Cd-MOF-74 & 110 & 17.9 & 350 & 34.0 & 48.1 & 0.71 & 91.2 \\
\hline Linker dhtp & - & - & 357 & 98.9 & 0.75 & - & - \\
\hline
\end{tabular}

${ }^{\mathrm{a}}$ Loss $2 / \mathrm{mol} \mathrm{MO}$, considering that $\mathrm{MO}(\mathrm{ZnO}$ or $\mathrm{CdO})$ is the unique residual phase after TGA treatment. 
Table 3. Textural properties extracted from the plots shown in Figure 5.

\begin{tabular}{|c|c|c|c|c|}
\hline \multirow[t]{2}{*}{ Sample } & \multicolumn{3}{|c|}{ Specific surface area $\left(\mathrm{m}^{2} \mathrm{~g}^{-1}\right)$} & \multirow{2}{*}{$\begin{array}{c}\text { Molar surface area } \\
\left(\mathrm{m}^{2} \mathrm{~mol}^{-1}\right)\end{array}$} \\
\hline & Total & Micropore & External & \\
\hline Zn-MOF-74 & 851 & 842 & 9 & 276,354 \\
\hline Cd-MOF-74 & 690 & 621 & 69 & 288,972 \\
\hline
\end{tabular}

${ }^{a}$ Specific surface area multiplied by the molecular weight of $\mathrm{Zn}_{2}(\mathrm{dhtp})\left(324.74 \mathrm{~g} \mathrm{~mol}^{-1}\right)$ or $\mathrm{Cd}_{2}(\mathrm{dhtp})$ $\left(418.80 \mathrm{~g} \mathrm{~mol}^{-1}\right)$. 